\title{
EDITORIAL \\ A smoker's paradox: does being a smoker really lead to a better outcome after aneurysmal SAH?
}

\author{
Kevin M. Cockroft, MD, MSc \\ Department of Neurosurgery, Penn State Hershey Medical Center, Hershey, Pennsylvania
}

$\mathrm{I}$ $\mathrm{N}$ this paper from Dasenbrock et al., the authors assess the impact of tobacco smoking on outcome after aneurysmal subarachnoid hemorrhage (SAH) by analyzing data from the National Inpatient Sample (NIS) and the National Surgical Quality Improvement Program (NSQIP) registry. ${ }^{3}$ The central finding of the study was that smoking was associated with superior outcomes after rupture on several measures, including overall clinical outcome as indicated by the NIS-SAH outcome measure (NIS-SOM). This finding was present in both the entire study population, as well as in poor-grade patients when considered separately. The finding also remained true over a range of smoking frequencies.

With administrative databases possessing many wellknown disadvantages, the authors took several steps to mitigate these limitations. Determining illness severity is often difficult and in this case the authors used the NISSAH severity scale, ${ }^{12}$ which correlates well with Hunt and Hess grade, to adjust for presenting severity. The score uses various related diagnostic and procedural codes, such as those for coma, hydrocephalus, hemiparesis, aphasia, and mechanical ventilation, to stratify patients. Similarly, the authors also used the NIS-SOM as their main clinical outcome measure. This externally validated end point defines outcome based on factors such as in-hospital mortality, discharge location, and tracheostomy/gastrostomy placement, with a poor NIS-SOM outcome closely mirroring a modified Rankin scale score of more than 3. ${ }^{12} \mathrm{By}$ using their own hospital's NSQIP data, the authors were also able to provide some verification of the accuracy of the data coding for smoking status. Finally, the authors used the statistical techniques of sensitivity analysis and propensity matching to assess the impact of a range of smoking frequencies on their outcome measures. While this analysis resulted in certain outcomes (such as in-hospital mortality, neurological complications, and delayed cerebral ischemia) no longer showing statistically significant differences, others measures remained significant, including extended hospitalization, discharge to institutional care, and poor NIS-SOM outcome.

The result is a robust, well-made case for a better outcome among smokers with aneurysmal SAH compared with nonsmokers. This is certainly a provocative and some might say paradoxical finding, but before we start encouraging all of our patients with aneurysms to take up smoking, there are some additional issues worth considering.

As many know, the prior literature has been mixed with regard to smoking and intracranial aneurysms. Some retrospective studies have suggested higher rates of vasospasm/delayed neurological deficits (DNDs) in smokers, ${ }^{7,8,13}$ while a report based on the "Get With The Guidelines" prospective database found better outcomes among smokers. ${ }^{1}$ There is also good evidence to indicate that smoking increases the risk of developing an intracranial aneurysm, as well as the risk of experiencing a subsequent rupture. ${ }^{4-6,9,11}$ Perhaps most interestingly, though, are two relatively recent retrospective studies that demonstrated improved outcomes in smokers with SAH who received nicotine replacement. ${ }^{2,10}$ Both studies found similar rates of radiographic vasospasm, while one reported similar rates of clinical ischemic events in the experimental and control groups and the other showed a lower rate among those receiving nicotine replacement therapy. Of note here, the current study was not able to directly investigate vasospasm/DNDs and had no way of determining whether or not smokers received nicotine replacement during their hospitalization.

Smokers as recorded by the NIS are a diverse group, which includes long-term and short-term smokers, as well as noncigarette smokers, all with different levels of use. Patients may also receive nicotine through other means, such as chewing tobacco, electronic cigarettes, and smok- 
ing cessation aids. As noted in the study, smokers tended to be much younger than nonsmokers. Although age was adjusted for in the multivariable analysis, the presence of additional unmeasured or unrecognized confounders potentially related to young age and or smoking status is always a concern in a retrospective study. Some potential covariates might include alcohol use (not abuse), nicotine replacement use, body mass index, and levels of physical activity.

Long-term outcome is another area to consider. As the authors point out, information on long-term outcome is lacking in the NIS, and discharge location may be a relatively crude measure of actual functional outcome. Will smoking hamper long-term brain recovery or will the comorbidities associated with smoking lead to other complications that impact long-term functional status?

From a practical standpoint, we are left with the question of where to go next. The finding that smokers may have a better outcome after aneurysmal SAH than nonsmokers may be interesting, but it still leaves us wondering what we as clinicians can do with this information in our treatment of such patients. An initial reaction might be to advocate for the use of nicotine supplementation. However, the present study does not really inform us in this regard. As the authors are quick to mention, tobacco smoke contains more than 4000 chemical components, of which nicotine may be the best studied, but arguably not necessarily the most important in this particular circumstance. With the rise in the use of electronic cigarettes, which have a more limited chemical composition, in the future it may be possible to perform a similar analysis to examine more directly the effects of nicotine itself. Importantly, the present study does not provide any information regarding the role of nicotine replacement in smokers, much less the role of nicotine supplementation in nonsmokers. If, as the present study suggests, smokers have better outcomes than nonsmokers, does adding nicotine supplementation further improve their outcome, as some other studies have suggested, or is their outcome already maximized by their prior exposure? Is this effect dose- or time-dependent? At present, the data do not appear to be sufficient to warrant a prospective randomized controlled trial (RCT) of nicotine therapy in aneurysmal SAH for nonsmokers and probably not even for smokers. However, multiinstitutional retrospective or prospective registry studies may confirm the findings of Dasenbrock et al. ${ }^{3}$ and help better frame the questions and design requirements for a subsequent prospective RCT.

https://thejns.org/doi/abs/10.3171/2016.12.JNS162628

\section{References}

1. Ali SF, Smith EE, Bhatt DL, Fonarow GC, Schwamm LH: Paradoxical association of smoking with in-hospital mortality among patients admitted with acute ischemic stroke. J Am Heart Assoc 2:e000171, 2013

2. Carandang R, Barton B, Rordorf G, Ogilvy C, Sims J: Nicotine replacement therapy after subarachnoid hemorrhage is not associated with increased vasospasm. Stroke 42:3080 3086, 2011

3. Dasenbrock HH, Rudy RF, Lai PMR, Smith TR, Frerichs KU, Gormley WB, et al: Cigarette smoking and outcomes after aneurysmal subarachnoid hemorrhage: a nationwide analysis. J Neurosurg [epub ahead of print October 27, 2017. DOI: 10.3171/2016.10.JNS16748]

4. Juvela S: Prevalence of risk factors in spontaneous intracerebral hemorrhage and aneurysmal subarachnoid hemorrhage. Arch Neurol 53:734-740, 1996

5. Juvela S, Hillbom M, Numminen H, Koskinen P: Cigarette smoking and alcohol consumption as risk factor for aneurysmal subarachnoid hemorrhage. Stroke 24:639-646, 1993

6. Knekt P, Reunanen A, Aho K, Heliövaara M, Rissanen A, Aromaa A, Impivaara O: Risk factors for subarachnoid hemorrhage in a longitudinal population study. J Clin Epidemiol 44:933-939, 1991

7. Krishnamurthy S, Kelleher JP, Lehman EB, Cockroft KM: Effects of tobacco dose and length of exposure on delayed neurological deterioration and overall clinical outcome after aneurysmal subarachnoid hemorrhage. Neurosurgery 61:475-481, 2007

8. Lasner TM, Weil RJ, Riina HA, King JT Jr, Zager EL, Raps $\mathrm{EC}$, et al: Cigarette smoking induced increase in the risk of symptomatic vasospasm after aneurysmal subarachnoid hemorrhage. J Neurosurg 87:381-384, 1997

9. Longstreth WT Jr, Nelson LM, Koepsell TD, van Belle G: Cigarette smoking, alcohol use, and subarachnoid hemorrhage. Stroke 23:1242-1249, 1992

10. Seder DB, Schmidt JM, Badjatia N, Fernandez L, Rincon F, Claassen J, et al: Transdermal nicotine replacement therapy in cigarette smokers with acute subarachnoid hemorrhage. Neurocrit Care 14:77-83, 2011

11. Teunissen LL, Rinkel GJ, Algra A, van Gijn J: Risk factors for subarachnoid hemorrhage: a systemic review. Stroke 27:544-549, 1996

12. Washington CW, Derdeyn CP, Dacey RG Jr, Dhar R, Zipfel GJ: Analysis of subarachnoid hemorrhage using the Nationwide Inpatient Sample: the NIS-SAH Severity Score and Outcome Measure. J Neurosurg 121:482-489, 2014

13. Weir BK, Kongable GL, Kassell NF, Schultz JR, Truskowski LL, Sigrest A: Cigarette smoking as a cause of aneurysmal subarachnoid hemorrhage and risk for vasospasm: a report of the Cooperative Aneurysm Study. J Neurosurg 89:405-411, 1998

\section{Disclosures}

The author reports no conflict of interest.

\section{Response}

\section{Hormuzdiyar H. Dasenbrock, MD, MPH, and Rose Du, MD, PhD}

Department of Neurosurgery, Brigham and Women's Hospital, Harvard Medical School, Boston, Massachusetts

Dr. Cockroft has written an insightful editorial about our article, and we appreciate his detailed analysis. As he has acknowledged, data were extracted in 5874 patients from across the US, and the goal of our study was to use a large national data set to evaluate the in-hospital outcomes of cigarette smokers who present with aneurysmal SAH, including mortality, neurological complications, tracheostomy or gastrostomy placement, length of hospital stay, discharge disposition, and poor outcome. However, Dr. Cockroft's editorial emphasizes the importance of critically analyzing population-based publications, and raises 3 important questions: 1) are the findings valid;2) are they biologically plausible; and 3) what are important future directions? 
One critical point that Dr. Cockroft has also underscored is that the while our study shows superior outcomes using many outcome measures for cigarette smokers (compared with nonsmokers) who present with aneurysmal SAH, we did not evaluate the natural history of unruptured cerebral aneurysms. Smoking is among the most potent risk factors for both cerebral aneurysm formation and rupture, ${ }^{1,2,5,8,9,11-13,15,17}$ and an incorrect conclusion from our study would be to suggest that smoking is beneficial to those with unruptured cerebral aneurysms. In fact, the analysis revealed that smokers sustained rupture at a younger age compared with nonsmokers. Given the potential associated morbidity of even those who sustain a good outcome after aneurysmal SAH, the younger age of rupture underscores the negative ramifications of cigarette smoking among those with cerebral aneurysms.

The first key question raised by our study is: are the findings of the study valid? The results of large population databases are viewed with trepidation by some authors, and thus this is an important question to address, by analyzing concordance with prior literature and the validity of the analysis.

The impact of smoking on complications and outcomes after aneurysmal SAH has been previously analyzed, and notably other publications have also reported superior outcomes among smokers, showing concordance of our findings with the work of other authors. Pobereskin evaluated the 30-day outcomes of 800 cases of aneurysmal SAH in two counties in the United Kingdom and the author reported a reduced risk of death at all end points evaluated for smokers. ${ }^{18}$ Additionally, 605 patients who presented to Columbia University Medical Center were evaluated retrospectively by Seder et al. to analyze the impact of nicotine replacement therapy. The primary goal of the paper was to compare smokers who received nicotine replacement therapy to smokers who did not receive nicotine replacement therapy, and nicotine replacement was associated with lower odds of death in this comparison. However, the mortality rate among smokers treated with nicotine replacement therapy was also lower compared with nonsmokers. ${ }^{19}$ While other authors have reported an association between smoking and delayed cerebral ischemia after aneurysmal SAH, ${ }^{6,14,16,20}$ these studies either did not evaluate neurological outcomes or mortality, ${ }^{20}$ or they were single-institution studies ${ }^{14,16}$ and thereby potentially underpowered, and none showed a negative effect on ultimate clinical outcome. Finally, while the validity of the statistical analyses is critical when assessing the findings of studies based on large-population databases, we appreciate Dr. Cockroft's assessment of our analysis as robust. In addition to performing multivariable regression analysis, we used multiple additional sensitivity analyses to reduce spurious findings due to confounding, including matched-pairs and propensity-adjusted analyses, as well as included a subgroup analysis of poor-grade patients, to reduce potential reporting bias.

A second key question that merits further comment is: are the clinical findings from this analysis biologically plausible? Cigarette smoke is a complex mixture of more than 4000 chemicals, and while the exact mechanism by which smoke promotes cerebral aneurysm formation is unclear, it may be partially attributable to chronic endothelial damage. ${ }^{47,10}$ Nevertheless, it is unclear if nicotine is the primary culprit for aneurysm formation; additionally, nicotine may have different effects in an acute and chronic setting. Caradang et al. have previously published a comprehensive review of the basic science literature evaluating the impact of nicotine on cerebral vasculature. These authors concluded that while nicotine has complex effects, it may have antiinflammatory, neuroprotective, and vasodilatory effects intracranially, which may be mediated by nitrous oxide and endothelium mechanisms using nicotinic acetylcholine receptors. ${ }^{3}$ Additionally, acute physiological effects of nicotine include an increase in heart rate, blood pressure, and cardiac output, similar effects to those that are desired during treatment of delayed cerebral ischemia. ${ }^{19}$ Thus, there remains a biologically plausible explanation whereby smoking could be associated with protective effects in the setting of acute SAH.

Finally, the most important question regarding the findings of the study is what are its consequences? Two prior retrospective, institutional studies have shown that nicotine replacement among smokers who present with aneurysmal SAH is associated with superior clinical outcomes compared with smokers treated without nicotine; one analysis reported superior Glasgow Outcome Scale score on discharge, ${ }^{3}$ and the other with reduced mortality 3 months posthemorrhage..$^{19}$ While the results of our study as well as these two prior retrospective studies are intriguing, a change in practice patterns would be significantly premature. Nevertheless, the results of our analysis, the studies that showed superior outcomes among smokers treated with nicotine replacement therapy, as well as two prior publications showing reduced mortality among smokers who sustained SAH, collectively suggest that smoking may provide a protective effect in the setting of acute aneurysmal SAH.

First, future basic science studies are needed to further understand the mechanisms for this relationship. Second, we agree with Dr. Cockroft that future clinical studies, including multiinstitutional studies, are needed to further confirm these findings, and provide a more nuanced assessment of the association of smoking with outcomesevaluating the length and dose of tobacco use-as well as control for additional potential confounding variables denoted by Dr. Cockroft, which were not available in prior studies, including our own. Moreover, other analyses of the association with smoking in the setting of CNS disease would be intriguing to evaluate if these differences are restricted to cerebrovascular disease, or observed in other intracranial pathology. However, ultimately an RCT may be needed to determine the effectiveness of nicotine replacement therapy in smokers in the setting of aneurysmal SAH, and perhaps in nonsmokers as well, depending on the results of studies in smokers.

\section{References}

1. Anderson CS, Feigin V, Bennett D, Lin RB, Hankey G, Jamrozik K: Active and passive smoking and the risk of subarachnoid hemorrhage: an international population-based case-control study. Stroke 35:633-637, 2004

2. Bonita R: Cigarette smoking, hypertension and the risk of 
subarachnoid hemorrhage: a population-based case-control study. Stroke 17:831-835, 1986

3. Carandang RA, Barton B, Rordorf GA, Ogilvy CS, Sims JR: Nicotine replacement therapy after subarachnoid hemorrhage is not associated with increased vasospasm. Stroke 42:30803086, 2011

4. Chalouhi N, Ali MS, Starke RM, Jabbour PM, Tjoumakaris SI, Gonzalez LF, et al: Cigarette smoke and inflammation: role in cerebral aneurysm formation and rupture. Mediators Inflamm 2012:271582, 2012

5. Davis MC, Broadwater DR, Amburgy JW, Harrigan MR: The clinical significance and reliability of self-reported smoking status in patients with intracranial aneurysms: a review. Clin Neurol Neurosurg 137:44-49, 2015

6. Dupont SA, Wijdicks EF, Manno EM, Lanzino G, Rabinstein AA: Prediction of angiographic vasospasm after aneurysmal subarachnoid hemorrhage: value of the Hijdra sum scoring system. Neurocrit Care 11:172-176, 2009

7. Etminan N, Beseoglu K, Steiger HJ, Hänggi D: The impact of hypertension and nicotine on the size of ruptured intracranial aneurysms. J Neurol Neurosurg Psychiatry 82:4-7, 2011

8. Feigin V, Parag V, Lawes CM, Rodgers A, Suh I, Woodward $\mathrm{M}$, et al: Smoking and elevated blood pressure are the most important risk factors for subarachnoid hemorrhage in the Asia-Pacific region: an overview of 26 cohorts involving 306,620 participants. Stroke 36:1360-1365, 2005

9. Feigin VL, Rinkel GJ, Lawes CM, Algra A, Bennett DA, van Gijn J, et al: Risk factors for subarachnoid hemorrhage: an updated systematic review of epidemiological studies. Stroke 36:2773-2780, 2005

10. Ho AL, Lin N, Frerichs KU, Du R: Smoking and intracranial aneurysm morphology. Neurosurgery 77:59-66, 2015

11. Juvela S, Hillbom M, Numminen H, Koskinen P: Cigarette smoking and alcohol consumption as risk factors for aneurysmal subarachnoid hemorrhage. Stroke 24:639-646, 1993
12. Juvela S, Lehto H: Risk factors for all-cause death after diagnosis of unruptured intracranial aneurysms. Neurology 84:456-463, 2015

13. Kim CK, Kim BJ, Ryu WS, Lee SH, Yoon BW: Impact of smoking cessation on the risk of subarachnoid haemorrhage: a nationwide multicentre case control study. J Neurol Neurosurg Psychiatry 83:1100-1103, 2012

14. Krishnamurthy S, Kelleher JP, Lehman EB, Cockroft KM: Effects of tobacco dose and length of exposure on delayed neurological deterioration and overall clinical outcome after aneurysmal subarachnoid hemorrhage. Neurosurgery 61:475-481, 2007

15. Lai LT, Morgan MK, Patel NJ: Smoking increases the risk of de novo intracranial aneurysms. World Neurosurg 82: 195e201, 2014

16. Lasner TM, Weil RJ, Riina HA, King JT Jr, Zager EL, Raps $\mathrm{EC}$, et al: Cigarette smoking-induced increase in the risk of symptomatic vasospasm after aneurysmal subarachnoid hemorrhage. J Neurosurg 87:381-384, 1997

17. Ortiz R, Stefanski M, Rosenwasser R, Veznedaroglu E: Cigarette smoking as a risk factor for recurrence of aneurysms treated by endosaccular occlusion. J Neurosurg 108:672675,2008

18. Pobereskin LH: Influence of premorbid factors on survival following subarachnoid hemorrhage. J Neurosurg 95:555559,2001

19. Seder DB, Schmidt JM, Badjatia N, Fernandez L, Rincon F, Claassen J, et al: Transdermal nicotine replacement therapy in cigarette smokers with acute subarachnoid hemorrhage. Neurocrit Care 14:77-83, 2011

20. Weir BK, Kongable GL, Kassell NF, Schultz JR, Truskowski LL, Sigrest A: Cigarette smoking as a cause of aneurysmal subarachnoid hemorrhage and risk for vasospasm: a report of the Cooperative Aneurysm Study. J Neurosurg 89:405-411, 1998 